\title{
Quantum Algorithm for High Energy Physics Simulations
}

\author{
Benjamin Nachman $\odot,{ }^{1, *}$ Davide Provasoli, ${ }^{1, \dagger}$ Wibe A. de Jong $\odot,{ }^{2, \S}$ and Christian W. Bauer ${ }^{1, \star}$ \\ ${ }^{1}$ Physics Division, Lawrence Berkeley National Laboratory, Berkeley, California 94720, USA \\ ${ }^{2}$ Computational Research Division, Lawrence Berkeley National Laboratory, Berkeley, California 94720, USA
}

(Received 14 September 2020; revised 2 December 2020; accepted 17 December 2020; published 10 February 2021)

\begin{abstract}
Simulating quantum field theories is a flagship application of quantum computing. However, calculating experimentally relevant high energy scattering amplitudes entirely on a quantum computer is prohibitively difficult. It is well known that such high energy scattering processes can be factored into pieces that can be computed using well established perturbative techniques, and pieces which currently have to be simulated using classical Markov chain algorithms. These classical Markov chain simulation approaches work well to capture many of the salient features, but cannot capture all quantum effects. To exploit quantum resources in the most efficient way, we introduce a new paradigm for quantum algorithms in field theories. This approach uses quantum computers only for those parts of the problem which are not computable using existing techniques. In particular, we develop a polynomial time quantum final state shower that accurately models the effects of intermediate spin states similar to those present in high energy electroweak showers with a global evolution variable. The algorithm is explicitly demonstrated for a simplified quantum field theory on a quantum computer.
\end{abstract}

DOI: 10.1103/PhysRevLett.126.062001

While quantum computers hold great promise for efficiently solving classical problems such as querying databases [1] or factoring integers into primes [2], their most natural application is to describe inherently quantum physical systems [3]. The most direct connection between quantum systems and quantum computers occurs for analog circuits that try to mimic the evolution of a Hamiltonian as closely as possible [4]. A promising alternative to analog circuits are digital quantum circuits, which use quantum algorithms to describe inherently quantum physical systems without directly implementing the system's Hamiltonian. However, many physical systems are too complex to fully model with a quantum circuit using near-future noisy intermediate scale devices [5]. This is true for a generic quantum field theory. While tools have been developed to model quantum field theories by discretizing spacetime [6] and even including continuous quantum numbers [7], the number of quantum bits required to compute any relevant scattering amplitude is impractically large. Results with simplified quantum field theories on a lattice are promising [8], but the full dynamics of high energy scattering processes are too complex for both lattice methods as well as traditional perturbation theory when the number of final state particles becomes too large.

Published by the American Physical Society under the terms of the Creative Commons Attribution 4.0 International license. Further distribution of this work must maintain attribution to the author(s) and the published article's title, journal citation, and DOI. Funded by SCOAP ${ }^{3}$.
A promising new avenue for quantum algorithms for quantum field theories is to use them only for the parts of the calculation that are computationally intractable using standard techniques.

It is well known that factorization theorems can be derived that separate shorter distance from longer distance physics [9-12]. The shortest distance physics of the hardscattering process is the most difficult part to simulate using lattice techniques (either using classical or quantum computers) and can in most cases be computed reliably using perturbative techniques. The longest distances correspond to hadronization effects for which often nonperturbative models are used to describe the physics. In between these two scales, one has to describe radiation occurring with soft and collinear divergences, which requires techniques beyond standard perturbation theory. A successful classical approach for simulating the dynamics of this final state radiation is known as the parton shower [13], which relies on reorganizing the traditional perturbative series about a fundamental coupling constant to instead expand around the collinear and soft limit of emissions $[14,15]$. This leads to different series expansio7ns where each term in the new series includes infinitely many terms from the original series expansion and is the basis of parton shower Monte Carlo (MC) programs [16-19], which are a key component of high energy quark and gluon scattering simulations. Parton shower models are implemented using classical Markov chain MC (MCMC) algorithms to efficiently generate high-multiplicity radiation patterns. This reliance on classical MCMC algorithms implies that several quantum interference effects need to be neglected. 
While many current analyses are nearly insensitive to such effects, future work will analyze the final state radiation in more detail and new studies will be enabled with calculations that include new quantum effects.

Our goal is to develop a quantum circuit describing the quantum properties of parton showers. In this work, we consider showers with quantum interferences from different intermediate particles, using a simplified model that captures these effects without having to introduce the full complexity of the standard model (SM). We consider showers that use a global evolution variable. While in classical showers the variable describing the scale of the shower evolution is continuous, it will be discretized for the quantum algorithm and at each step an emission can occur or not. We will show that a classical MCMC is not able to capture the important quantum interference effects in this model, and that a full classical calculation scales exponentially with the number of steps. [There are efficient algorithms to account for spin correlations in quantum chromodynamics [20-23], but these do not apply to our model or more generally to any model such as SU(2) where the emission probability depends on the spin [24]. We restrict ourselves to showers using a global evolution variable, where the evolution of a given parton in the event depends on all other partons. Most modern parton showers such as dipole and $p_{T}$ ordered showers have this global evolution.] The proposed quantum algorithm will be able to sample from the full probability distribution in polynomial time.

To begin, consider a simple quantum field theory, with two types of fermion fields, $f_{1}$ and $f_{2}$, interacting with one scalar boson $\phi$ governed by the following Lagrangian:

$$
\begin{aligned}
\mathcal{L}= & \bar{f}_{1}\left(i \not \partial+m_{1}\right) f_{1}+\bar{f}_{2}\left(i \not \partial+m_{2}\right) f_{2}+\left(\partial_{\mu} \phi\right)^{2} \\
& +g_{1} \bar{f}_{1} f_{1} \phi+g_{2} \bar{f}_{2} f_{2} \phi+g_{12}\left[\bar{f}_{1} f_{2}+\bar{f}_{2} f_{1}\right] \phi .
\end{aligned}
$$

The first three terms in Eq. (1) describe the kinematics of the fermions and scalar while the latter three terms govern their interactions. In particular, the collinear dynamics of the theory are that the fermions can radiate scalars $\left(f_{i} \rightarrow f_{j} \phi\right)$ and scalars can split into fermion pairs $\left(\phi \rightarrow f_{i} \bar{f}_{j}\right)$. These couplings of fermions to scalar bosons occur in the Higgs sector of the SM, and it has been demonstrated that the final state collinear radiation at high energy can be written in terms of a parton shower [25,26]. This model can contain important quantum interference effects when all couplings are nonzero, since the unobserved intermediate state of the fermions can be a superposition of $f_{i}$ for $i \in\{1,2\}$.

In the limit $g_{12} \rightarrow 0$ one can derive an efficient MCMC method for calculating high-multiplicity cross sections. This is performed by introducing four splitting functions, two for a fermion radiating a scalar $\left[P_{i \rightarrow i \phi}(\theta)=g_{i}^{2} \hat{P}_{f}(\theta)\right]$ and two for the scalar splitting into fermions $\left[P_{\phi \rightarrow i i}(\theta)=g_{i}^{2} \hat{P}_{\phi}(\theta)\right]$, where $\theta$ is the scale at which the splitting occurs and $\hat{P}(\theta)$ encodes the energy scale dependence of the emission probability (we suppress additional splitting variables, which can be handled classically as mentioned in the Supplemental Material [27]). There are many formally equivalent definitions of the scale; here we use a common choice: the opening angle of the emission with respect to the emitter. In addition to the splitting functions, another important quantity is the no-branching probability (Sudakov factor):

$$
\Delta_{i, k}\left(\theta_{1}, \theta_{2}\right)=\exp \left[-g_{i}^{2} \int_{\theta_{1}}^{\theta_{2}} d \theta^{\prime} \hat{P}_{k}\left(\theta^{\prime}\right)\right] .
$$

The Sudakov factor encapsulates the virtual (and unresolved real) contributions and is responsible for the reorganization of the perturbation series ("resummation") mentioned above. The Sudakov factor and splitting function satisfy the unitarity relation

$$
\Delta_{i, k}\left(\theta_{1}, \theta_{2}\right)+g_{i}^{2} \int_{\theta_{1}}^{\theta_{2}} d \theta \hat{P}_{k}(\theta) \Delta_{i, k}\left(\theta, \theta_{2}\right)=1 .
$$

A classical parton shower would then efficiently sample from the cross section using a Markov chain algorithm by generating one emission at a time, conditioned on the last emission. Once the evolution variable $\theta$ is discretized, emissions can only happen at these discrete values, and we assume that the discretization is fine enough that at most one emission can happen at each step. This means that at a given step $n$ in $\theta$ there are at most $n$ particles and the probability that none of them radiate or split is $\prod_{j=1}^{N} \Delta_{i_{j}, k_{j}}$. If something does happen at a given step, the probabilities are proportional to the appropriate splitting function. The smallest scale accessible to the shower is denoted by $\epsilon$.

When $g_{12}>0$, there are now multiple histories with unmeasured intermediate fermion types which contribute to the same final state. Therefore, the above MCMC is invalid because one must include all possible histories and cannot condition on a given state. Including all of the interference effects requires accounting for all histories at the amplitude level and only computing probabilities at the end of the evolution. When the $g_{12} \ll 1$, the evolution is dominated by a single emission, which can be properly treated using a density matrix formalism [25], where each splitting function is represented through a splitting matrix. For example, the fermion splitting matrix is $P_{i \rightarrow j \phi}(\theta)\left|f_{i}\right\rangle\left\langle f_{j}\right|$ (outer product of a ket and bra gives a matrix). When there is more than one emission during the evolution, this matrix formalism is insufficient and one must compute the full amplitude for which there are $\mathcal{O}\left(2^{N}\right)$ possible histories.

We propose an efficient solution by keeping track of amplitudes and not probabilities using a quantum computer. A quantum circuit implementing the quantum final state radiation algorithm for one of $N$ steps is given by the following diagram: 


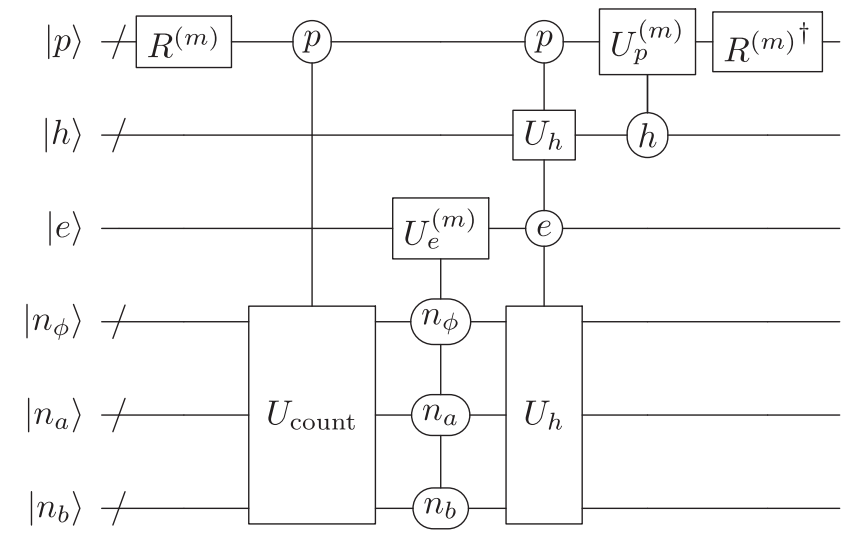

The circuit calls for six registers, which are detailed in the Supplemental Material [27] and summarized in Tables I and II. The initial state of $|p\rangle$ consists of $n_{I}$ particles (which can be fermions or bosons) in the $f_{1 / 2}$ basis. One starts by rotating this initial particle state from the $f_{1 / 2}$ basis to a diagonal $f_{a / b}$ basis, using a simple unitary $R^{(m)}$ operation. Then, a series of operations evolving the particle states are applied: the number of particles of each type are counted $\left(U_{\text {count }}\right)$, Sudakov factors are used to determine if an emission occurred $\left(U_{e}^{(m)}\right)$, given an emission, a particular particle is chosen to radiate and branch $\left(U_{h}\right)$, and the resulting particle state is updated $\left(U_{p}^{(m)}\right)$. Finally, the state is rotated back to the $f_{1 / 2}$ basis through the $R^{(m) \dagger}$ operation. This process is repeated for all of the $N$ steps.

Performing the evolution in the $f_{a / b}$ basis and then rotating to the $f_{1 / 2}$ basis, creates interferences between equivalent final states which had different intermediate fermions. One event is generated by measuring all of the qubits after the final rotation back to the $f_{1 / 2}$ basis. By repeating the entire process, we can generate a large number of events which we can then use to compute physical observables for our theory. The algorithm presented can be simplified significantly if the subset of qubits representing the history register $|h\rangle$ can be measured at the end of each step. This fixes the total number of particles as well as the total number of bosons. The number of standard quantum gates (single qubit and CNOT gates) required at

TABLE I. All of the registers in the quantum circuit with the number of qubits they require for $N$ steps and $n_{I}$ initial particles. The symbol $\lceil\ldots\rceil$ denotes the ceiling function.

\begin{tabular}{lcc}
\hline \hline Register & Purpose & Number of qubits \\
\hline$|p\rangle$ & Particle state & $3\left(N+n_{I}\right)$ \\
$|h\rangle$ & Emission history & $N\left\lceil\log _{2}\left(N+n_{I}\right)\right\rceil$ \\
$|e\rangle$ & Did emission happen? & 1 \\
$\left|n_{\phi}\right\rangle$ & Number of bosons & $\left\lceil\log _{2}\left(N+n_{I}\right)\right\rceil$ \\
$\left|n_{a}\right\rangle$ & Number of $f_{a}$ & $\left\lceil\log _{2}\left(N+n_{I}\right)\right\rceil$ \\
$\left|n_{b}\right\rangle$ & Number of $f_{b}$ & $\left\lceil\log _{2}\left(N+n_{I}\right)\right\rceil$ \\
\hline \hline
\end{tabular}

each step is discussed in the Supplemental Material [27] and summarized in Table II with and without the repeated measuring of the history register. Comparing the scaling of the quantum algorithm with an efficient classical algorithm, the quantum algorithm outperforms the classical algorithm once the number of emitted particles exceeds $\mathcal{O}(10)$, if the history register is measured after each step. Without this repeated measurement, the number of steps required for the quantum algorithm to beat the classical one depends on the size of the coupling constants $g_{1,2}$ as the classical scaling goes with the number of fermions and not the (much) larger number of steps.

The practical challenge with above circuit is that it requires more connected qubits and operations than are currently available in state-of-the-art hardware. In order to show an implementation of our algorithm, we therefore consider a special case that is amenable to measurement on existing technology. This special case ignores the $\phi \rightarrow f \bar{f}$ splitting (naturally suppressed in gauge theories, but not in the scalar-only theory), ignores the running coupling, and has only a single fermion (possibly in a superposition) as the initial state. This results in a much simpler circuit since there is only one fermion, but an arbitrary number of scalars. A decomposition of the resulting circuit into single qubit and CNOT gates requires $n_{\text {gates }}=12 N+2$. This model is, however, still sufficiently complex that the classical MCMC described earlier fails to capture important quantum effects when $g_{12} \neq 0$. (We should note that this simplified problem does allow for an efficient classical algorithm [28]. However, the classical MCMC algorithm that is required for the full problem still fails to give the correct prediction.)

Figure 1 presents the normalized differential cross sections for the logarithm of the largest emission angle (a),(c) as well as the number of emissions (b),(c) for both classical simulations and calculations, quantum simulators [29], and chip experiments of public and Q Hub member quantum chips through cloud access on the IBM Quantum Experience. All cases are started from the initial state containing a single $f_{1}$ fermion, and use the splitting function $P_{f}(\theta)=g^{2} /(4 \pi) / \theta$. We choose $\epsilon=10^{-3}$ and have $N$ equidistant steps between $\epsilon$ and 1. The data of experimental measurements shown in Fig. 1 were collected on the IBM Q Johannesburg chip. This quantum computer has twenty qubits, and to restrict the gate depth and hardware fidelity challenges we choose to simulate $N=4$ steps. The 4-step circuit on 5 qubits requires 48 gate operations, of which 17 are 2-qubit operations. Details of the experiments, including measurement corrections are discussed in the Supplemental Material [27], which includes Refs. [30-33]. In addition to presenting the simplified model with both quantum hardware and simulations, Fig. 1 also shows a simulation with the full model (including $\phi \rightarrow f \bar{f}$ ) for two steps. 
TABLE II. List of the circuit operations with the number of standard gates required for given numbers of steps assuming $n_{I}=1$. Further details about the calculations involved and the counting of the number of gates can be found in the Supplemental Material [27]. The third column provides the scaling assuming that classical registers could be used to store the history qubit at each step. This is not implemented in the algorithm shown in Fig. 1, but may be possible on near-term hardware.

\begin{tabular}{|c|c|c|c|}
\hline \multirow[b]{2}{*}{ Operation } & \multicolumn{2}{|c|}{ Scaling } & \multirow{2}{*}{$\begin{array}{c}\text { Number of gates (default algorithm) } \\
N=4\end{array}$} \\
\hline & Default algorithm & Measure $|h\rangle$ & \\
\hline Count particles $\left[U_{\text {count }}\right]$ & $N \ln N$ & $N \ln n_{f}$ & $4.93 \times 10^{2}$ \\
\hline Decide emission $\left[U_{e}\right]$ & $N^{4} \ln N$ & $N n_{f} \ln n_{f}$ & $9.29 \times 10^{3}$ \\
\hline Create history $\left[U_{h}\right]$ & $N^{5} \ln N$ & $N n_{f}^{2} \ln n_{f}$ & $1.96 \times 10^{5}$ \\
\hline Adjust particles $\left[U_{p}\right]$ & $N^{2} \ln N$ & $N n_{f} \ln n_{f}$ & $5.01 \times 10^{3}$ \\
\hline Classical algorithm & \multicolumn{2}{|c|}{$N 2^{n_{f} / 2}$} & \\
\hline
\end{tabular}

When interference effects are turned off $\left(g_{12}=0\right)$, we find excellent agreement for all observables between both the classical and quantum simulator results as well as the quantum computer measurements. For $g_{12}=1$ the spectra are shifted to the right, leading to more emissions and at larger angles. For all quantum simulations the fraction of events with no emissions [first bin in (b) and (d)] agree separately for each value of $g_{12}$. This is because the simulation is started with a single fermion state, where the splitting $\phi \rightarrow f \bar{f}$ is irrelevant. For a higher number of emissions, the $\phi \rightarrow f \bar{f}$ splitting affects the distribution, and in particular lowers the fraction of events with a single emission.

The experimental data points obtained running the 48 operation simulation on the IBM Q Johannesburg quantum computer are in agreement with the quantum simulator results, clearly showing the role of interference when the interaction is turned on (from $g_{12}=0$ to $g_{12}=1$ ). Some differences can be observed between the quantum simulator and the actual quantum computer experiment, which can be


(c)
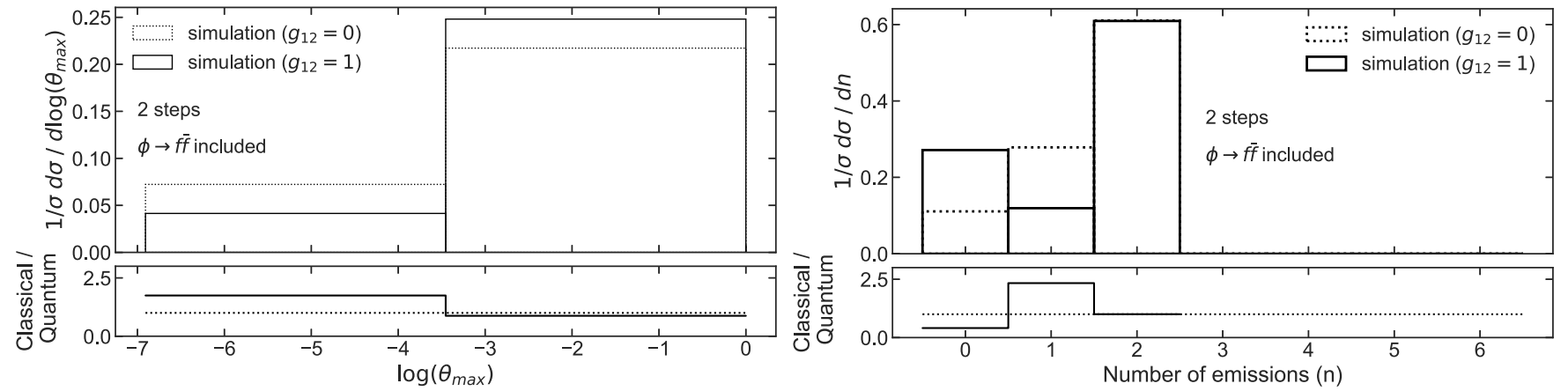

FIG. 1. The normalized differential cross section for $\log \theta_{\max }$ (a),(c) and the number of emissions (b),(d). Interference effects are turned on $\left(g_{12}=1\right)$ and off $\left(g_{12}=0\right)$, where the classical simulations and calculations are expected to agree with the quantum simulations and measurements. The top plots (a),(b) show results for the case where $\phi \rightarrow f \bar{f}$ is excluded as this can be run on current quantum hardware. The bottom plots (c),(d) include the $\phi \rightarrow f \bar{f}$ with fewer steps to reduce the computational complexity. The ratio plots compare the $g_{12}=0$ and $g_{12}=1$ simulation. Over $10^{5}$ events contribute to each line and the statistical uncertainties are therefore negligible. Quantum measurements are corrected for readout errors, as described in the Supplemental Material [27]. Running coupling effects are ignored for simplicity. 
attributed to the noise present in the existing hardware. These results are an important and first-of-its-kind experimental proof of principle for the algorithm and are the first step in the realization of a larger and more precise calculations on future hardware.

Future extensions of the algorithm can bring it closer to the SM. To include the full three-dimensional kinematics of parton splittings, one would need to augment the procedure to sample momentum fractions and azimuthal angles. It may be possible to achieve this with a hybrid quantumclassical approach as the probability distribution factorizes. The current model includes all of the discrete quantum numbers of the Higgs sector of the SM with one fermion. Adding additional fermions is algorithmically simple and is a linear computational cost. A further extension to the SU(2) component of the SM would be able to use nearly the same algorithm, but with more fermions and additional bosons to represent the $W^{ \pm}$and $Z$ bosons. While the numerical implications of such electroweak showers with multiple bosons may be small at previous and current colliders, such calculations may be important for future colliders as well as indirect dark matter search experiments with ultrahigh energy particles [34] with a Higgs or full $\mathrm{SU}(2)$-like structure. Further extensions to the strong force SU(3) may be possible, but are more complicated because of the important role of low energy and not just collinear radiation. There is also an extensive literature on approximate amplitude-based approaches using classical methods [35-41], which may also have potential synergy with quantum algorithms in the future.

With improved quantum hardware beyond the current noisy intermediate scale devices [5], our algorithms will be able to produce calculations that are currently not possible with classical devices. The richness of quantum phenomena in high energy physics makes them an excellent test bed for studying the power of quantum algorithms. By focusing on final state radiation, quantum algorithms may be able to provide key insight into the dynamics of quantum field theories underlying the laws of nature.

We thank A. Ryd for useful discussions on the treatment of spin correlations in classical MCMC programs. This work was supported by the U.S. Department of Energy (DOE) under Contract No. DE-AC02-05CH11231, through the Office of High Energy Physics Quantum Information Science Enabled Discovery (QuantISED, KA2401032) Program. This research used resources of the OakRidge Leadership Computing Facility, which is a DOE Office of Science User Facility supported under Contract No. DEAC05-00OR22725.

*bpnachman@lbl.gov

†davideprovasoli@lbl.gov

*cwbauer@lbl.gov

${ }^{\S}$ WAdeJong@lbl.gov
[1] L. K. Grover, in Proceedings of the Twenty-Eighth Annual ACM Symposium on Theory of Computing, arXiv:quant-ph/ 9605043.

[2] P. W. Shor, SIAM J. Sci. Stat. Comput. 26, 1484 (1997).

[3] R. P. Feynman, Int. J. Theor. Phys. 21, 467 (1982).

[4] I. M. Georgescu, S. Ashhab, and F. Nori, Rev. Mod. Phys. 86, 153 (2014).

[5] J. Preskill, Quantum 2, 79 (2018).

[6] S. P. Jordan, K. S. M. Lee, and J. Preskill, Science 336, 1130 (2012).

[7] K. Marshall, R. Pooser, G. Siopsis, and C. Weedbrook, Phys. Rev. A 92, 063825 (2015).

[8] E. A. Martinez et al., Nature (London) 534, 516 (2016).

[9] J. C. Collins and D. E. Soper, Annu. Rev. Nucl. Part. Sci. 37, 383 (1987).

[10] G. F. Sterman, in QCD and Beyond. Proceedings of the Theoretical Advanced Study Institute in Elementary Particle Physics, TASI-95, Boulder, 1995, pp. 327-408, arXiv:hep$\mathrm{ph} / 9606312$.

[11] R. L. Jaffe, in The Spin Structure of the Nucleon. Proceedings of the International School of Nucleon Structure, 1st Course, Erice, Italy, 1995, pp. 42-129, arXiv:hep-ph/ 9602236.

[12] C. W. Bauer, S. Fleming, D. Pirjol, I. Z. Rothstein, and I. W. Stewart, Phys. Rev. D 66, 014017 (2002).

[13] M. Tanabashi et al. (Particle Data Group), Phys. Rev. D 98, 030001 (2018).

[14] G. F. Sterman and S. Weinberg, Phys. Rev. Lett. 39, 1436 (1977).

[15] L. G. Almeida, S. D. Ellis, C. Lee, G. Sterman, I. Sung, and J. R. Walsh, J. High Energy Phys. 04 (2014) 174.

[16] T. Sjostrand, S. Mrenna, and P. Z. Skands, J. High Energy Phys. 05 (2006) 026.

[17] M. Bahr et al., Eur. Phys. J. C 58, 639 (2008).

[18] T. Gleisberg, S. Hoeche, F. Krauss, M. Schonherr, S. Schumann, F. Siegert, and J. Winter, J. High Energy Phys. 02 (2009) 007.

[19] A. Buckley et al., Phys. Rep. 504, 145 (2011).

[20] I. G. Knowles, Nucl. Phys. B310, 571 (1988).

[21] I. G. Knowles, Comput. Phys. Commun. 58, 271 (1990).

[22] J. C. Collins, D. E. Soper, and G. F. Sterman, Adv. Ser. Dir. High Energy Phys. 5, 1 (1989).

[23] P. Richardson, J. High Energy Phys. 11 (2001) 029.

[24] P. Richardson and S. Webster, Eur. Phys. J. C 80, 83 (2020).

[25] J. Chen, T. Han, and B. Tweedie, J. High Energy Phys. 11 (2017) 093.

[26] C. W. Bauer, D. Provasoli, and B. R. Webber, J. High Energy Phys. 11 (2018) 030.

[27] See Supplemental Material at http://link.aps.org/ supplemental/10.1103/PhysRevLett.126.062001 for details of the proposed quantum algorithm and its simulation on IBMQ quantum computers.

[28] D. Provasoli, B. Nachman, W. A. de Jong, and C. W. Bauer, Quantum Sci. Technol. 5035004 (2020).

[29] IBM Research, Qiskit, an open-source computing framework (2018).

[30] M. A. Nielsen and I. L. Chuang, Quantum Computation and Quantum Information, 10th ed. (Cambridge University Press, New York, NY, 2011). 
[31] B. Nachman, M. Urbanek, W. A. de Jong, and C. W. Bauer, npj Quantum Inf. 6, 84 (2020).

[32] E. F. Dumitrescu, A. J. McCaskey, G. Hagen, G. R. Jansen, T. D. Morris, T. Papenbrock, R. C. Pooser, D. J. Dean, and P. Lougovski, Phys. Rev. Lett. 120, 210501 (2018).

[33] IBM Research, Qiskit Ignis.

[34] C. Blanco, J. P. Harding, and D. Hooper, J. Cosmol. Astropart. Phys. 04 (2018) 060.

[35] Z. Nagy and D. E. Soper, J. High Energy Phys. 09 (2007) 114.

[36] Z. Nagy and D. E. Soper, Phys. Rev. D 99, 054009 (2019).
[37] S. Plätzer, M. Sjodahl, and J. Thorén, J. High Energy Phys. 11 (2018) 009.

[38] S. Platzer and M. Sjodahl, J. High Energy Phys. 07 (2012) 042.

[39] R. Ángeles Martínez, M. De Angelis, J. R. Forshaw, S. Plätzer, and M. H. Seymour, J. High Energy Phys. 05 (2018) 044.

[40] J. R. Forshaw, J. Holguin, and S. Plätzer, J. High Energy Phys. 08 (2019) 145.

[41] J. Isaacson and S. Prestel, Phys. Rev. D 99, 014021 (2019). 